\title{
$\mathrm{X}$.
}

Ans dem pathologischen Institut zu Heidelberg.

\section{Ueber die Muskelatrophie bei der cerebralen Hemiplegie.}

\author{
Von \\ Dr. A. Steiner.
}

Die Thatsache, dass central gelähmte Muskeln nur wenig oder gar nicht in ihrer Ernährung leiden, ist seit Langem bekannt und diagnostisch verwerthet. Und doch ist unverhältnissmässig viel Zeit dariuber hingegangen, bis das Bewusstsein derselben einem grösseren Kreise lebendig wurde. Selbst im Beginn der zweiten Hälfte unseres Jahrhunderts findet man in wissenschaftlichen Arbeiten die Atrophie nach Lähmungen in einer Weise dargestellt, die an Unklarheit nichts zu wünschen übrig lässt. Man kann daher erst recht nicht hoffen, etwaige Ausnahmen von der Regel schon in der älteren Literatur verzeichnet zu finden. Vielmehr macht der Schwund cerebral gelähmter Muskeln da, wo ich ihn zuerst erwähnt finde ${ }^{1}$ ), ganz den Eindruck eines höchst gleichguiltigen und zufälligen Nebenumstandes, der in anderen Fällen ebenso gut versehwiegen, wie hier gerade angeführt werden konnte.

Gewöhnlich wird Todd ${ }^{2}$ ) als der Erste genannt, der sich eingehender mit den Zuständen der Muskeln bei Hemiplegischen be-

1) M. Hall (Krankheiten des Nervensystems. Leipzig 1842): „bei einer chronischen Hemiplegie mit bedeutender Abmagerung der gelähmten Mus keln". Was die Literaturangaben dieser Arbeit betrifft, so babe ich natürlich Alles beigezogen, was ich finden lonnte, von den älteren auch die Hauptwerke durchgesehen, soweit sie mir erreichbar waren. Zu meinem grossen Bedauern konnte ich namentlich die italienische Literatur nicht in dem Masse berücksichtigen, wie sie es verdiente; auch die französische dürfte noch eines und das andere enthalten. Wo ferner die Atrophie nur gelegentlich unter anderem Titel erwähnt war, blieb ihre Entdeckung natürich dem Zufall überlassen, so dass vielleicht auch hier noch einige modificirende Thatsachen zu finden wären. Aber für eine darauf gerichtete Arbeit ist kein Ende abzusehen.

2) Clinical Lectures on Paralysis. London 1854. 2. ed. 1856. 
schäftigt hat. Ich finde aber, dass schon 1848, also mindestens gleichzeitig und dem Datum der Veröffentlichung nach sogar noch einige Jahre früher Helfft ${ }^{1}$ ) dieselben ebenfalls behandelt und im Wesentlichen zu denselben Ergebnissen kommt. Uns interessirt von seinem Schema hier nur die zweite Gruppe, wo die Muskeln „von vornherein in hohem Grade welk und schlaff sind und schnell abmagern". Diese „Hemiplegie mit schlaffen Muskeln" bildet die erste Klasse der Toddschen Eintheilung. Todd's Bedeutung für unsere Frage wird anch von $\mathrm{Brissaud}$ in seiner historischen Betrachtung gebührend hervorgehoben. Die Lectüre seiner ausgezeichneten „Vorlesungen“, besonders der darin mitgetheilten Fälle, in deren einem die Atrophie so hochgradig war, dass der Humeruskopf aus der Gelenkpfanne herabsank, zeigt, dass Todd so weit gekommen war, wie man eben ohne Kenntniss der absteigenden Degeneration und ohne genaue mikroskopische Untersuchung kommen konnte. Auch Le ubuscher ${ }^{2}$ ) spricht, wenn auch bei Weitem nicht mit der Todd'sehen Gründlichlzeit, an verschiedenen Stellen von der Muskelatrophie bei der cerebralen Hemiplegie. Etwa ein Decennium später haben Cornil ${ }^{3}$ ) und Bou chard ${ }^{4}$ ) die atrophischen Muskeln und ihre Nerven bei einer Anzahl Hemiplegischer untersucht und in jenen einfache und degenerative Atrophie, in diesen Bindegewebswucherung nachgewiesen. Aber, was uns heute kaum begreiflich scheinen will, das Ritckenmark wurde nicht berïcksichtigt und damit der Sehlïssel zur Lösung des Räthsels nicht gefunden. $\mathrm{Cbarcot}$ blieb es vorbehalten, theils selbst, theils durch seine Schüler die Ursache dieser posthemiplegischen Atrophie aufzudecken, als welche in allen darauf untersuchten Fällen atrophische Processe im betreffenden Vorderhorn gefunden wurden, hervorgerufen durch Uebergreifen der absteigenden Degeneration der Pyramidenbahn anf die graue Substanz. ${ }^{5}$ ) Diese bis dahin gewöhnlich

1) Neuropath. Beobachtungen. Casper's med. Wochenschrift. 1848. S. 822. Eine etwa gleichzeitige hierhergehörige Beobachtung Romberg's (,Lehrbuch der Nervenkrankheiten $1850^{\circ}$ ) citirt Carrieu. Ich muss gestehen, dass es mir nicht geglückt ist, sie aufzufinden.

2) Pathologie und Therapie der Gehirnkrankheiten. Berlin 1854. Was sich etwa in späteren Hand- und Lehrbüchern über diesen Gegenstand findet, werde ich nicht weiter aufzählen.

3) Gaz. médic. de Paris 1864. No. 11. 4) Ebenda. No. 28.

5) Charcot, Leçons sur les malad. du syst. nerv. I. Paris 1874. - Leçons sur les localisat. cérébr. I. Paris 1876. - Carrieu, Des amyotrophies spinal second. Thèse. Montpellier 1875. - Pitres, Gazette des hôpitaux u. Arch. de physiol. 1876. - Brissaud, De l'atrophie musculaire dans l'hémiplégie, Revue mens, de méd. 1879. p. 616. 
für unmöglich gehaltene Complication der absteigenden Degeneration war vereinzelt auch sonst beobachtet und in ihrer Bedeutung erkannt worden ${ }^{1}$, und jetzt ging man im Gegentheil so weit, sie für ihr eigentliches und regelrechtes Ende zu erklären (Brissaud). Namentlich durch die Arbeit Brissaud's war das Bild dieser, wie ich sie der Kürze wegen nennen will, „spinalen" Form der posthemiplegischen Muskelatrophie einstweilen zum Abschluss gebracht, und konnte die spätere Zeit nur noch weitere casuistische Beiträge liefern, deren Zabl eine auffallend geringe genannt werden mag. ${ }^{2}$ )

Während man noch damit beschäftigt war, diese spinale Form, so gut es gehen wollte, mit den herrschenden Anschaungen in Einklang zu bringen, boten sich aufmerksamen Beobachtern neue Rätbsel. 1879 berichtete Senator ${ }^{3}$ ) über einen Fall von Gehirnabseess mit schubweise eintretender Hemiplegie und einer sehr schnell (binnen 11 Tagen) sich entwickelnden Abmagerung des befallenen Armes, während bei der Section weder absteigende Degeneration, noch Vorderhornalterationen gefunden wurden. In den letzten Jahren worden dann so viele mit diesem identische Fälle mitgetheilt, dass auch die "cerebrale" Form der posthemiplegischen Muskelatrophie, d. h. die ohne Veränderungen in den Vorderhörnern, mit oder ohne absteigende Degeneration, als eine zwar seltene, aber sicher bestehende Folgeerscheinung einer Hirnerkrankung angesehen werden musste. Je ärmer wir an Thatsachen waren, desto mehr bemühte man sich, durch $\mathrm{Hy}-$ pothesen diese sonderbare Erseheinung sich zurechtzulegen. Allein die Ueberraschungen hatten noch nicht einmal ein Ende. Seitdem man die Hysterie genauer kannte, wusste man auch von dem Vorkommen einer hysterischen Hemiplegie. Für die Diagnose derselben war, wie für eine hysterische Affection tiberhaupt, das Fehlen jeder trophischen Störung von fundamentaler Wichtigkeit. Aber auch hier zeigte sich bald, dass keine Regel obne Ausnahme bleibt. Zuerst finde ich bei Benedikt4) beginnende Atrophie als bysterische Trophoneurose erwähnt. Ziemlich lange danach hat dann $\mathrm{K} a \mathrm{k}_{\mathrm{k}}$ off $\mathrm{f}^{5}$ ) und etwas später

1) Hallopeau, Arch. génér. de méd. 1871/72. 1872. p. 74. - Leyden, Klinik der Rückenmarkskrankheiten. Berlin 1874/76. Bd. II. S. 304.

2) Bastian (Common forms of paralysis from brain disease), Lancet 1874. II. p. 405. - Putzel, New York med. Record. 1882. - Joffroy et Achard, Arch. de méd. expériment. III. p. 780.1891.

3) Berl. klin. Wochenschrift. 1879. Ich nenne diesen Fall als den ersten, weil die Atrophie hier zuerst gebührend hervorgehoben wurde.

4) Wien. med. Wochenschrift. 1863. Nr. 30.

5) Zur Differentialdiagnose der u. s. w. Hemianästhesie. Diss. Halle 1884. 
Charcot, resp. Babinski ${ }^{1}$ ) eine Reibe von hysterischen Mono- und Hemiplegien mit Muskelatrophie mitgetheilt. Seitdem ist noch eine Anzahl solcher Fälle bekannt geworden, die ich hier nicht weiter aufzuzählen brauche.

Wie man leicht sieht, ist mit der Atrophie bei der hysterischen Hemiplegie die Reihe der Möglichkeiten zu Ende. Denn wir haben jetzt die posthemiplegische Muskelatrophie unter folgenden Verhältnissen:

Gehirn.

1. nachweisbar verändert

2. nachweisbar verändert

3. nach weisbar verändert
4. nicht nach- weisbar ver- ändert

Pyramidenbahn. nachweisbar verändert nachweisbar verändert
nicht nach- weisbar ver- ändert. nicht nachweisbar verändert

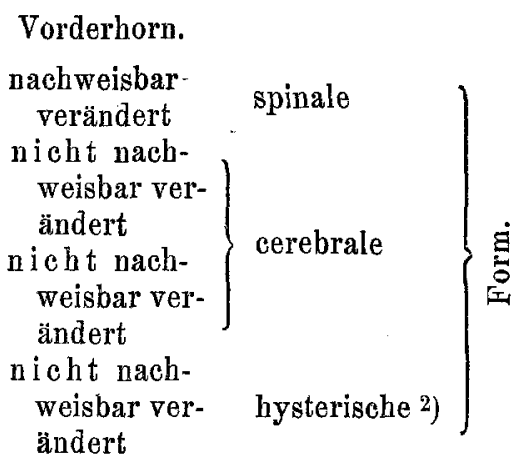

Nach diesem kaum zu umgehenden Ueberblick beginnen wir die Beschreibung der cerebralen Form der posthemiplegischen Muskelatrophie, als welche unser eigentliches Thema bildet, mit der Aufzählung ibrer Literatur. Auf einen Missstand, der sich dabei störend

1) Charcot, Leẹ. sur les mal. du syst. nerv. III. Anhang. - Babinski, Arch. de neurol. XII. 1886.

2) Von der corticomusculären Leitungsbahn wäre jetzt nur noch ein Stück übrig, dessen Degeneration auch Muskelatrophie erzeugen könnte. In der That hat Déjé rine (Soc. de biol. 27. VII. 1889) über einige Fälle von posthemiplegischer Muskelatrophie referirt, als deren Ursache er eine centralwärts an Intensität abnehmende Degeneration der peripheren Nerven betrachtet wissen will. Die Vorderhörner waren nicht verändert. Ueber das Verhalten der Pyramidenbahn ist in dem Auszug der Gaz. hebd. 1889 leider nichts gesagt. Déjéri ne hält die Atrophie spinalen Ursprungs für sehr selten und die Degeneration der peripheren Nerven für ihre gewöhnliche Ursache. Aber wenn man auch der Auffassung D éjérine's, die durch die Art der Ausbreitung der Degeneration freilich wenig gestützt wird, beipflichten wollte, so hätte man doch für das Verständniss der Frage damit gar nichts gewonnen. Denn die Muskeln und Nerven falien hier völlig unter einen Begriff, den des Endorgans, und wenn man bisher fragte: wie kommt es, dass die Muskeln bei Hemiplegischen hie und da atrophiren? so würde man eben jetzt fragen: wie kommt es, dass bei ihnen hie und da die Nerven degeneriren? Der Vollstăndigkeit halber sei noch bemerkt, dass in nevester Zeit auch eine hysterische Muskelatrophie ohne Lähmung beschrieben wurde (Gilles de la Tourette, Nouv. Iconogr. de la Salpetrière. II. 1889; nach Schmidt's Jahrb. 226. S. 24). 
bemerkbar macht, habe ich schon oben hingedeutet, dass nämlich besonders die älteren Arbeiten in ihren Ueberschriften auch nicht im Mindesten eine etwa bemerkte Atrophie verrathen and ihre Entdeckung also nur einem glibeklichen Zufall gelingen kann. So ging es mit den Fällen Senator's, Burresi's, Cantani's und Rosenthal's. ${ }^{1}$ ) Ein anderes Uebel ist die ungleiche Ausfihrlichkeit der Mittheilungen. Von den drei Fällen, die Babinski anfiuhrt, sind nur von einem ein paar kümmerliche Daten zu verwerthen, von den zwei anderen wissen wir nur, dass sie gesehen wurden.

Was die Auswahl des Materials anlangt, so bleiben hier alle nicht erwachsenen, also auch 2 von den seither immer zusammen besprochenen Patienten unberïcksichtigt. Denn bei Kindern gehören Differenzen im Volumen u. s. w. gesunder und central gelähmter Glieder zu den gewöhnlichen Vorkommnissen, mögen sie nun bald nach $\mathrm{Be}$ ginn der Erkrankung oder erst nach Monaten und Jahren eintreten ${ }^{2}$ ), und wenn dabei auch neben der Störung des Wachsthums eine wirkliche Atrophie eine grössere Rolle spielt, als Manche zugeben wollen, so ist doch eben diese Concurrenz zweier verschiedener nicht scharf za trennender Momente so hinderlich, dass wir lieber, einstweilen wenigstens, auf die ganz eindentigen Beobachtungen an Erwachsenen uns beschränken. So wissen wir bis jetzt von 21 Fällen, von 2 (Babinski) allerdings nur, dass sie existirten, von 2 anderen ( $\mathrm{Can}$ tani, Rosenthal) kaum viel mehr, so dass noch 17 übrig sind, an denen wir unsere Betrachtungen anstellen können. Dies sind der Reihe nach:

1 Burresi (Tuberculosi delia zona eccitabile dell' emisfero cerebrale destro). Lo sperimentale. 1877. I. p. 248.

1 Senator (Zur Diagnose der Hirnerkrankungen). Berl. klin. Wochenschr. 1879.

1) Silvestrini (Le paralisi nei loro rapporti colla atrofia dei muscoli. Tesi. Padova 1875) spricht bei Patella (l. c.) von 2 Fällen Cantani's und Rosenthal's, intracranielle Tumoren, die Brücke comprimirend, mit Atrophie im Gefolge der dadurch erzeugten Lähmung. Leider war es mir unmöglich, Genaneres über dieselben zu erfahren.

2) Vgl, u. A, Taylor (On unilateral atrophy and spasm), Guy's Hosp. Rep. XXIII. 1878. - Putzel (l. c.) in der an den Vortrag sich anschliessenden Discussion. - Henoch, Vorlesung über Kinderkrankheiten. Berlin 1890. - Förster, Ueber trophische Störungen bei Lähmungen. Deutsche med. Wochenschr. 1880. S. 671 (rasch eintretende Atrophien bei hemiplegischen Kindern). Analog den letzteren sind ein von Senator (Charité-Annalen XIII. S. 323) und ein von Nothnagel (Zur Diagnose der Sehhügelerkrankungen), Zeitschr. f. klin. Med. XVI. 1889 im Nachtrag mitgetheilter Fall. Ob Wachsthumshemmung oder Atrophie, mag vorläufig noch unerörtert bleiben. Vgl. S. 297 Anm. 
3 Patella (Delle atrofie musculari secondarie). Padova 1886.

1 Babinski (Soc. de biol.) referirt: Neur. Centralbl. 1886.

$2 \mathrm{Q}$ aincke (Ueber Muskelatrophie bei Gehirnerkrankung). Deutsches Arch. f. klin. Med. XXXXII. Bd. S. 492. 1887.

1 Borgherini (Ueber die frühzeitige Muskelatrophie bei der cerebralen Lähmung). Deutsches Arch. f. klin. Med. XXXXV. Bd. S. 371. 1889.

$1 \mathrm{Klippel}$ (Hémiplégie gauche avec troubles trophiques ete.). Bull. de la soc. anat. de Paris. LXIV. p. 649. 1889.

2 Eisenlohr (Muskelatrophie bei Gehirnherden). Neurolog. Centralbl. 1890.

1 Borgherini (Ueber einen Fall frühz. Maskelatrophie u. s. w.). Ebenda. S. 545.

2 Roth et Mouratow (Contribut. ̀̀ l'étude de la pathol. des hémisphères cérébraux). Moscou 1890. (Arch. de neurol. Vol. XXI. p. 296.) Vgl. anch Muratoff, Vortrag auf dem 4. Congress russischer Aerzte. Tageblatt des Congresses und Referat im Arch. de neurol. XXI. p. 461.

1 Darkschewitsch (Ein Fall von frühzeitiger Muskelatrophie bei einem Hemiplegiker). Neurol. Centralbl. 1891. \$. 622.

$1 \mathrm{Kramer}$ (Beitrag zur Lehre von der Jakson'schen Epilepsie und der cerebral bedingten Muskelatrophie). Jahrb. f. Psych. X. S. 91. 1891.

Hieran reiht sich als Nr. 18 ein auf der hiesigen medicinischen Klinik behandeiter Patient mit linksseitiger Hemiplegie und Atrophie des Armes, von dem die uns hauptsächlich interessirenden Daten mit denen der 17 anderen in einer Tabelle (Seite 286 u. 287) zusammengestellt sind, während die etwas eingehendere Beschreibung hier folgt.

Krankengeschichte.

Joh. E., 72 J. alt, wurde am 8 . Februar 1890 in die medicinische Klinik anfgenommen, nachdem er vom 15. December 1889 an in poliklinischer Behandlung gewesen. Ats den poliklinischen Aufzeichnungen war zu entnehmen, dass Pat. früher stets gesund, nicht luetisch und kein Trinker war. Doch hatte er seit Sommer 1889 hie und da auf ganz kurze Zeit nicht mehr sprechen können und einen nach links geneigten unsicheren Gang bekommen. Auch über etwas Herzklopfen klagte er. Am 14. December 1889 fiel er plötzlich bewasstlos zu Boden, kam zwar bald wieder zu sich, konnte aber Arm und Bein links nicht mehr bewegen. Am anderen Tage untersucht, zeigte er Ptosis und Facialislähmung links, sowie spastische Lähmung der linken Extremitäten. Die Zunge war frei, Pupillen beiderseits gleich, 


\begin{tabular}{|c|c|c|c|c|c|}
\hline $\mathrm{Nr}$. & Autor & 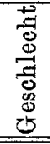 & 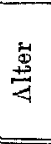 & Art des Leidens & Beginn der Atrophie \\
\hline 1 . & Patella 1.c. & M. & 21 & Fractur des Os par. dex. & $\begin{array}{l}\text { Nach } 2 \text { Monaten be- } \\
\text { trächtl, Atrophie. }\end{array}$ \\
\hline 2. & $\begin{array}{l}\text { Borgherini, D. } \\
\text { A. f. kl. Med. }\end{array}$ & M. & 24 & $\begin{array}{l}\text { Hämorrhagie in die rechte Strei- } \\
\text { fensehhügelgegend? }\end{array}$ & Am 3. Tag. \\
\hline 3. & Quincke l. c. & M. & 25 & $\begin{array}{l}\text { Leptomening. syphil. gyri centr. } \\
\text { dextr. utriusque. }\end{array}$ & Nach 3 Wochen. \\
\hline 4. & Senator l.c. & M. & 30 & Abscess in der linken Hemisphäre. & Nach 11 Tagen $1-2 \mathrm{Cm}$. \\
\hline 5. & Burresil. c. & W. & 30 & $\begin{array}{l}\text { Zahlreiche Tuberkel und sklero- } \\
\text { tische Herde in d.r. Hemisphäre. }\end{array}$ & $\begin{array}{l}\text { Arm unbestimmt, im Bein } \\
24 \text { Tagenach d. Lähmung. }\end{array}$ \\
\hline 6. & Eisenlohr l. c. & M. & 34 & $\begin{array}{l}\text { Abscess in Nucl. caudat. u. Reg. } \\
\text { subthal. sinistr. }\end{array}$ & Bemerkt nach 2 Monaten. \\
\hline 7. & $\begin{array}{l}\text { Rothu. Maratow } \\
\text { l.c. }\end{array}$ & M. & 35 & Gliosarcom. gyri centr. dextr. & $?$ \\
\hline 8. & Patella $1 . \mathrm{c}$ & W. & 36 & Embolie der 1. Art. foss. Sylvii. & $\begin{array}{l}\text { Nach } 2 \text { Monaten bis } \\
\text { 3,5 Cm. }\end{array}$ \\
\hline 9. & Quineke l.c. & M. & 39 & $\begin{array}{l}\text { Glioma gyri centr. dextr. utrius- } \\
\text { que. }\end{array}$ & Nach 4 Wochen. \\
\hline 10. & Kramer l.c. & M. & 40 & $\begin{array}{l}\text { Leptomening. chronica atrophica } \\
\text { cerebri. }\end{array}$ & $?$ \\
\hline 11. & $\begin{array}{l}\text { Darkschewitsch } \\
\text { l. c. }\end{array}$ & M. & 43 & Haemorrh. corpor. striati sinistri. & Nach 4 Wochen. \\
\hline 12. & Patella l.c. & W. & 45 & Gliom. corticis dextr. & $?$ \\
\hline 13. & $\begin{array}{l}\text { Borgherini, N. } \\
\quad \text { C. } 1890 .\end{array}$ & M. & ธั0 & $\begin{array}{l}\text { Metast. Sarkom der linken Cen- } \\
\text { tralwindung. }\end{array}$ & Nach 2 Monaten. \\
\hline 14. & Klippel l. c. & W. & 63 & $\begin{array}{l}\text { Hämorrh. in Caps. intern. und } \\
\text { Thal. optic. reehts. }\end{array}$ & ? \\
\hline 15. & Eisenlohr l. o. & W. & 70 & $\begin{array}{l}\text { Erweichungsherd der rechten } \\
\text { Hemisphäre. }\end{array}$ & Nach 4 Wochen. \\
\hline 16. & Unser Fall & M. & 72 & $\begin{array}{l}\text { Hämorrh. in Thal. optic. u. Caps. } \\
\text { inter. dextr. }\end{array}$ & Nach 8 Wochen $3 \mathrm{Cm}$. \\
\hline 17. & Roth u. Muratow & $?$ & $?$ & $\begin{array}{l}\text { Apoplekt. Herd in der Gegend der } \\
\text { 3. Stirnwindung u. Insel rechts. }\end{array}$ & $?$ \\
\hline 18. & Babinski l.c. & $?$ & ? & $\begin{array}{l}\text { Erweichungsherd im Centr. ovale } \\
\text { dextr. }\end{array}$ & $?$ \\
\hline
\end{tabular}

Sehnenreflexelinks gesteigert, Hantreflexedagegen herab. gesetzt, Sensibilität stark verringert. Leichte Trübung des Bewusstseins. Auf den Lungen Alles normal, Herzschlag sehr unregelmässig, Gefässe starr und hart, Incontinentia vesicae, Temp. normal.

In den folgenden Wochen ging die Lähmung des Beines so weit zurück, dass nur noch eine mässige Parese bestehen blieb, die Ptosis verlor sich völlig. Das Sensorium wurde wieder frei, doch war Pat. i mmer schläfrig und, wie sich jetat zeigte, dement (war er wahrscheinlich schon vor dem Anfall). Neu hinzu kamen Schmerzen, die bald in den ganzen Körper, 
Ueber die Muskelatrophie bei der cerebralen Hemiplegie.

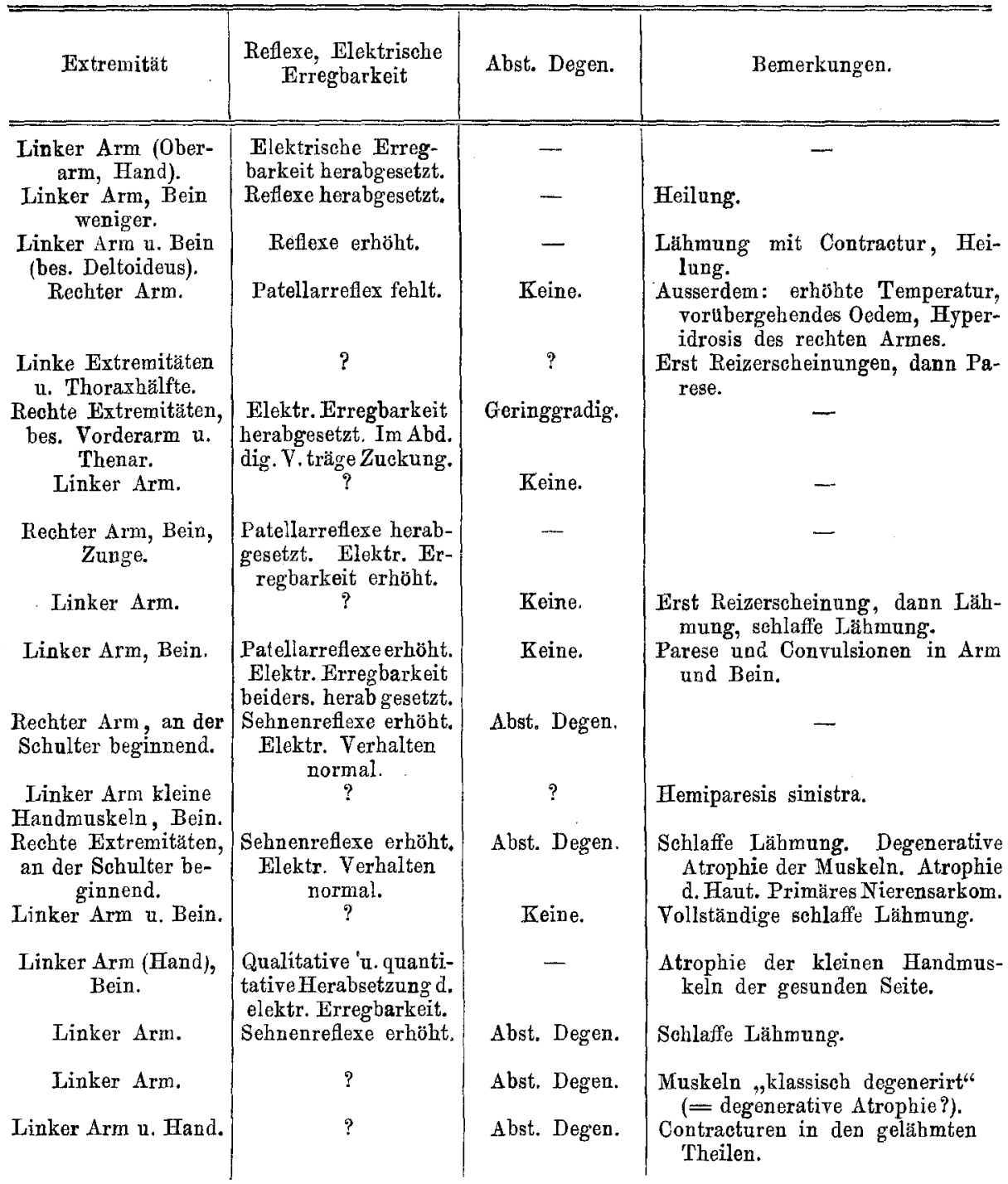

bald in die 4 Extremitäten, bald nur in die gelähmten localisirt wurden. Allmählich entwickelte sich Decubitus am Kreuzbein. Diesen Angaben entsprechend constatirte man bei der Aufnahme in die Klinik:

Cerebrale Hemiparese links (VII, Arm, Bein). Im Bein ziemlich starke Muskelspannungen, Arm dagegen völlig schlaff und an Umfang 2-3 $\mathrm{Cm}$. weniger messend, als der rechte. Patellarreflex beiderseits erhöht. Spannungen im rechten Adductorengebiet. Pupillen reagiren gut. Dementia senilis. Unregelmässiger schneller Puls. Systolisches Geräusch an der Herzspitze bei starker 
Herzthätigkeit. Decubitus. ${ }^{1}$ ) Die Neigung zu seblafen nahm zu. Vom 13. Februar an bewusstlos. Cheyne-Stokes'sches Athmen stellte sich ein, rechts hinten unten zeigten sich die physikalischen Symptome einer hypostatischen Pneumonie, der Puls wurde immer schneller und schwächer, und am 19. Februar 1890 trat der Tod ein. Die am gleichen Tage im pathologischen Institut vorgenommene Section ergab: Hypertrophie and Myodegeneration des Herzens, Pleuropnenmonie beider Unterlappen, leichte Schrumpfniere, Prostatahypertrophie. Im Gehirn: Arteriosklerose, hämorrhagischer Herd, vorwiegend im rechten Sehhügel, kleine ältere Erweichungsherdehen in der linken Hemisphäre. Gehirn und Rückenmark wurden sofort in Mü̈ller'sche Flüssigkeit gebracht und die genauere Untersuchung auf später verschoben.

Nach der Härtung zeigt sich am Rückenmark schon dem blossen Auge sehr deutlich die absteigende Degeneration des linken Pyramidenseitenstrangs als eine gelbliche Stelle anf dunkelm Grunde, ebenso im oberen Halsmark die der rechten Pyramidenvorderstrangbabn.

Die mikroskopische Untersuchung wurde an nach der Weigert'schen und Pal'schen Methode behandelten, ebenso an mit Borax- oder Lithioncarmin oder Nigrosin gefärbten Schnitten vorgenommen. Die Freud'sche Goldfärbung versagte leider. Zunächst war auffallend, dass an gleichviel mit welcher Methode gefärbten Präparaten die degenerativen Veränderungen makroskopisch kaum noch zu erkennen waren. Aber auch noch bei sehwacher Vergrösserung gaben wenigstens die nach Pal und Weigert gefärbten Schnitte lange nicht so schöne Bilder, wie man sie nach der blossen Betrachtung der unbehandelten Stiicke erwarten durfte. Erst bei sehr sorgfältigem Zusehen fand man anch an solchen Schnitten, dass den degenerirten Partien entsprechend die Färbung nicht ganz so scharf und intensiv war wie normaler Weise.

Was nun die Verbreitung der secundären Degeneration angeht, so zeigte der linke Pyramidenseitenstrang dieselbe in seiner ganzen Länge von unten nach oben in zunehmendem Maasse. Das Eingehen auf mikroskopische Einzelheiten kann ich lassen, da nichts von dem Gewöhnlichen Abweichendes sich zeigte. Ausser dem linken Pyramidenseitenstrang war, im unteren Brustmark beginnend und ebenfalls nach oben an Intensität zunehmend, die Degeneration im rechten Pyramidenvorderstrang, besonders an Carmin- und Nigrosinpräparaten schön zu sehen. Der rechte Pyramidenseitenstrang zeigte im Lenden- und unteren Dorsalmark spärlicher, im oberen Brust- und Halsmark reichlicher über seinen Querschnitt verstreute degenerirte Fasern und Faserlïcken, ein Befund, der von Interesse ist, weil er das anatomische Substrat für die gesteigerten Sehnenreflexe und Muskelspannungen in der rechten Unterextremität abgiebt. Im linken Pyramidenvorderstrang konnten höchstens im

1) Ueber die Art der Facialislähmung (ob nur willkürlich oder auch reflectorisch) Genaueres festzustellen, war bei der klinischen Untersuchung wegen der Demenz und des heruntergekommenen Kräftezustandes des Pat. nicht mehr gut möglich. Auch auf die Prüfung des elektrischen Verhaltens musste aus gleichem Grunde verzichtet werden. 
oberen Halsmark ganz vereinzelte Fasern als krankhaft veränderte angesprochen werden. Sonst bot derselbe ein durchaus normales Verhalten. Die soitlicheGrenzschicht der grauen Substanz war besonders links, weniger rechts, in den oberen Abschnitten mehr, im Dorsalmark weniger, der Intensität der Veränderungen in den Pyramidenbahnen entsprechend degenerirt. Von Brissaud (l. c.) wird darauf hingewiesen, dass anf der Seite, wo die Pyramidenbahn degenerirt, auch die Proc. reticul. und die in sie eingestrenten Ganglienzellen schwinden (die Atrophie der letateren hat v. Monakow, Neurol. Centralbl. 1885. S. 69 für die Katze als Regel beschrieben), Fürstner (Neurol. Centralbl. 1889, Arch. f. Psych. und Nervenkrankh. XXIII. Heft 1), erwähnt Gestaltsveränderungen der Vorderhörner infolge des Untergangs der eintretenden Nervenfasern. Von all dem zeigte sich in unserem Fall nichts. Man wird aber darin nichts Ungewöhnliches finden; denn wenn auch bei absteigender Degeneration diese Veränderungen nach den angeführten Gewährsmänmern mit einer gewissen Regelmässigkeit einzutreten scheinen, so ist doch zu bedenken, dass dazu vor Allem längere Zeit gehört, und dass unser Pat. den Anfall nur um etwa 9 Wochen überlebt hat. Besondere Aufmerk samkeit wurde natürlich dem Verhalten der Ganglienzellen in den Vorderhörnern und den vorderen $W$ urzeln zugewendet. Nirgends konnte man so beständige Unterschiede zwischen beiden Seiten finden, dass die eine zuletzt hinter der anderen zurückgestanden wäre in der Zahl der dem einzelnen Vorderhorn zugetheilten Zellen. Aber auch an den Zellen selbst berechtigte nichts, sie für atrophisch oder sonst krankhaft verändert zu erklären. Die vorderen Wurzelfasern, soweit sie đurch die weisse Sub. stanz verliefen ebensowohl, wie auf dem Querschnitt als ausgetretene vordere Wurzeln, waren nicht im Geringsten verändert. Namentlich in den am meisten in Betracht kommenden und besonders zahlreichen Schnitten aus der Halsanschwellung bot sich nichts Verdächtiges.

Als Alterserscheinungen waren anzusehen die stärkere Pigmentirung: einzelner Zellgruppen, die grosse Zahl von Amyloidkörpern und arteriosklerotische Veränderungen an den das Rückenmark versorgenden Gefässen, besonders der Art. spin. ant. Das Bindegewebe war nicht vermehrt, nicht einmal im Bereich der stärksten Degeneration.

Im verlängerten M a rk ist ausser der Degeneration der Pyramidenbahn nichts bemerkenswerth. Am stärksten ergriffen ist die rechte, aber auch die linke geht nicht frei aus. Konnte nun die Untersuchung des Rückenmarks anfänglich die Vermuthung nahe legen, dass es sich bei dieser Betheiligung der eigentlich gesunden Seite um die öfter beobachtete Folge verschiedener Durchkreuzungsverhältnisse der Pyramidenbahnen handelte, so musste man jetzt diese Vorstellung fallen lassen und an der absteigenden Degeneration der rechten Pyramidenbahn Veränderungen in der linken Hemisphäre Schuld geben, auf die wir nachher noch zu sprechen kommen. Die Degeneration der Pyramidenbahn in der bis jetzt beschriebenen Weise war zu verfolgen bis zu den hinteren Vierhügeln. Auf die Untersuchung der Hirnschenkel musste leider verzichtet werden, weil durch ein Unglück beim Conserviren gerade dieser Abschnitt in seinen wichtigsten Theilen zu Grunde gegangen war. Für die Sache ist das glücklicher Weise ja belanglos. Im Gebiet des rechten hinteren Vierhügels fand sich ein rom 
Aquaed. Sylv. nur durch eine $1 / 2-1 / 3 \mathrm{Mm}$. breite Brücke getrennter, von oben innen nach unten aussen längsovaler und in dieser Richtung etwa $2 \mathrm{Mm}$. langer, $1 \mathrm{Mm}$. breiter hämorrhagischer Herd. Die Länge desselben (Gehirn - Rückenmark) mochte vielleicht etwas über $1 \mathrm{Mm}$. betragen; derselbe lag völlig innerhalb der den Aquaed. auskleidenden grauen Substanz. Der eigentliche Vierhügelkern war intact, auch die in einiger Entfernung vorbeiziehenden III und VWurzeln wurden nicht berührt. Dagegen sind die feinen markhaltigen Fasern in der umgebenden grauen Substanz, besonders nach oben und innen von dem Herd ganz geschwunden oder nur noch spurweise vorhanden. Eine weitergehende Wirkung lässt sich nicht nachweisen, denn einige Faserlücken im hinteren Längsbündel auf der Seite des Herdes sind sicher die Folge ungleichmässiger Conservirung, so verführerisch es auch sein mag, dieselben mit der Hämorrhagie, speciell mit dem Faserschwund in deren Umgebung in Verbindung zu bringen. Anzunehmen, dass dieser Herd irgend welche Symptome gemacht habe, hat man keinen Grund. Den unsicheren Gang des Pat. im letzten halben Jahre zu erklären, haben wir in der linken Hemisphäre so viel Veränderungen, dass es nicht nöthig ist, auf diese kleine Verletzung zuräckzugreifen. Von Sehstörungen, die man noch am ehesten hätte erwarten dürfen, ist nichts bemerkt. Es ist aber auch aus anatomischen Gründen geradezu unwahrscheinlich, dass diese Hämorrhagie überhaupt vor der in den Thal. opt. stattgefunden haben solle. Die Untersuchungen über die Blutgefässvertheilung im centralen Nervensystem haben ergeben, dass Vierhügel und Thal. opt. zusammen von einem Gefäss der Art. cerebr. post. versorgt werden, und nichts ist natürlicher sich vorzustellen, als dass beide zugleich von der Circulationsstörung betroffen wurden. Eben daraus erlklärt ja auch Nothnagel die ausserordentliche Seltenheit, resp. das völlige Fehlen isolirter Ausfallsherde in den Vierhügeln, und unser Fall ist wieder ein sprechender Beweis für die Richtigkeit dieser Anschaung.

Der hämorrhagische Herd der rechten Hemisphäre nimmt, wie schon bemerkt, vorwiegend den Sehhügel ein. 'Der hinterste Abschnitt desselben bleibt frei. Dafür greift hier die Erweichnng auch auf die äussere Wand des Ventrikelhorns über, die einige Millimeter tief zerstört ist. Die Ausdehnung in sagittaler Richtung beträgt etwa 2,5 Cm. Medial gegen den Ventrikel zu ist nur noch eine ziemlich dünne, manchmal ganz minimale Schichte übrig geblieben, die nur am vorderen Pol des Herdes wieder an Breite gewinnt. Nach aussen reicht er mit einer leicht convexen Begrenzungslinie ein Stückchen in die innere Kapsel hinein, ebenso in seiner Mitte, als an der Stelle seiner grössten Ausdehnung, nach unten. Es sind also alle drei gewöhnlich am Thal. opt. unterschiedenen Kerne zerstört, mit Ausnahme eines Theiles des Nucl. ant. und med. am vorderen Ende und des hinteren Pulvinarabsehnittes; Theile der inneren Kapsel sind theils primär getroffen, theils secundär erweicht. Der Faserschwund in der Caps. intern. ist ibberall ein sehr bedeutender, schon makroskopisch erkennbarer.

In der linken Hemisphäre fanden sich eine Reihe versehieden grosser Herde an verschiedenen Stellen. Zunächst sassen zwei kleine, strichförmige von 2 , resp. $3 \mathrm{Mm}$. Länge mitten im Sehhügel dieser Seite, entsprechend der Höhe der Comm. mollis. In deren Umgebung sind die markhaltigen Fasern in erheblichem Maasse gesehwunden. Der anliegende 
Abschnitt der inneren Kapsel zeigt keine Veränderungen. Ein anderer Herd findet sich in der lateralen Wand des Hinterhorns, bohnengross, mit der Längsaxe in frontaler Richtung stehend, ein weiterer, streifenförmiger, im Gebiet des oberen Drittels der Centralwindungen, ausserdem noch einige Herdchen in der Inselrinde. Diese Herdchen, besonders die im Sehhügel, der Insel und den Centralwindungen, dürften zur Erklärung der geringen absteigenden Degeneration der linken Pyramidenbahn im verlängerten Mark ausreichen.

Eine mit Rücksicht anf die senile Demenz vorgenommene Untersuchung der Hirnrinde ergab: beträchtliche Arteriosklerose der Pialund Rindengefässe, Verminderung der markhaltigen Fasern. Die Ganglienzellen, besonders die motorischen der Centralwindungen, berechtigten ihrem Aussehen nach keineswegs zur Annahme degenerativ-atrophischer Zustände. Allerdings zeigten, namentlich im Stirnhirn, einige derselben körnigen Zerfall in verschiedenen Abstufungen, so dass manchmal nur der Kern mit einem kleinen Hänfchen körniger Masse übrig war. Solche Bilder fanden sich aber auch in einem zum Vergleich herangezogenen normalen Gehirn and dürften wohl am ersten als Folge der Conservirung angesehen werden. Amyloidkörperchen, deren reichliches Vorkommen Kostjurin (Neurol. Centralbl. 1886. S. 132) als Symptom der senilen Veränderungen anführt, wurden nicht gefunden.

Eine Untersuchung der Muskeln und Nerven konnte wegen Mangels an Material nicht vorgenommen werden.

Als nicht erwachsen bleiben von den seither immer zusammen besprochenen ausser Betracht der Kranke von Glicky (D. A. f. klin. Med. 1875. 15 Jahre) und einer von Quincke (14 Jahre). Die Tabelle (Seite 252 u. 253) giebt von den übrigen das Wichtigste und erleichtert die Uebersicht.

Aus dieser kleinen Zahl unter sich wieder ungleichwerthiger Beobachtungen irgend allgemeinere Schlitsse zu ziehen, würde man vielleicht am besten ganz unterlassen. Indessen hat Borgherini seiner Zeit schon aus 7 Fällen das Wesentliche der cerebralen Atrophie zu charakterisiren gesucht, so dass uns jetzt bei mehr als doppelt so vielen fast die Verpflichtung obliegt, diese Borgherini'schen Ausführungen auf ihre Richtigkeit zu prüfen und, wenn nöthig, zu modificiren.

Unsere meisten Patienten sind Männer, entsprechend der grösseren Häufigkeit der verursachenden Erkrankungen beim männlichen Geschlecht.

Was das Alter betrifft, so constatirte Borgherini die vorwiegende Betheiligung der Jugend. Das erscheint auch jetzt noch der Hauptsache nach richtig und, wenn man sich an die oben erwähnte Neigung des Kindesalters zu derartigen Atrophien erinnert, fast selbstverständlich. Es haben aber doch die neueren Fälle 
(Borgherini II, Klippel, Eisenlohr II u.s. w.) gezeigt, dass die Erscheinung auch dem böheren und selbst dem Greisenalter nicht fremd ist. Im Uebrigen sind die Zahlen viel zu klein für speciellere Angaben (von 16 mit bekanntem Alter liegen zwischen 21-30:5, $31-40: 5,41-50: 3,51-60: 0,61-70: 2$, über $71: 1$ ).

Der kürzeste Zeitraum zwischen Beginn der Erkrankung und Eintritt der Atrophie betrug einmal 2 Tage und nie mehr als 8 Wochen, wobei zu bemerken ist, dass nach dieser äussersten Zeit die Differenz im Umfang schon $2-3 \mathrm{Cm}$. ausmachte, der Schwund also schon ziemlich viel früher begonnen haben musste. Auf jeden Fall unterscheidet sich die cerebrale Form zeitlich so viel von der spinalen, dass man wohl ein Recht hat, beide als Früh - und Spätform auseinanderzuhalten. Freilich fragt sich, $o b$ der Unterschied immer so gross bleiben wird, wie er jetzt ist. Der Nachweis der absteigenden Degeneration ist allmählich immer kürzere Zeit nach Eintritt der Läsion gelungen, und so wäre es denkbar, dass auch ibr Uebergreifen auf die Vorderhörner bei aufmerksamer Beobachtung einmal früher als seither gesehen wird.

Höchst merkwürdig ist das Ueberwiegen der linken Körperseite (iiber 2/3), weil es sich nicht nur bei der cerebralen, sondern auch bei der spinalen und der hysterischen Form findet. Von den spinalen Fällen Brissaud's sitzen nämlich links 5 von 7 , von den hysterischen Babinski's 5 von 6 . Die erste Frage ist natürlich die, ob nicht überhaupt die Hemiplegien links häufiger sind, als rechts. Aus verschiedenen Statistiken ergiebt sich am Ende eine kleine Ueberzahl zu Gunsten der linken Körperseite. So findet Gintrac (nach Hammond ${ }^{1}$ ) unter 369 Fällen von hämorrbagia eerebri die Mehrzahl rechts, Durand-Fardel (ebenda) mit 117 Fällen allerdings links (rechts 49, links 57), wofür aber Drozda ${ }^{2}$ ) auf die rechte Hemisphäre 50,6, auf die linke 45,5 Proe. herausrechnet. Scheinen also auch wirklich die rechtsseitigen Hämorrhagien etwas häufiger zu sein, so ist doch der Unterschied ein so geringer, dass er bei Weitem nicht an die oben angeführten heranreicht. Nur die hysterischen Lähmungen zeigen eine ganz auffallende Vorliebe für die linke Körperseite, wie schon Mesnet ${ }^{3}$ ) hervorgeboben hat. Wenn nun auch anfänglich diese Thatsache theils angezweifelt, theils für rein zufällig erklärt wurde, so liessen sich doch bald grösseren Zusammenstel-

1) Diseases of the nerrous System. London 1881.

2) Statistisches über Haemorrh. cerebr. Wien. med. Presse. 1880. 10 u. 11.

3) Etudes des paralysies hystériques. Thèse. Paris 1852 (citirt nach Schmidt's Jahrbüchern). 
lungen, wie z. B. der Brown-Séquard's, gegeniuber solche Einwände nicht mehr gut machen; vielmehr ist man genöthigt, die vorwiegende Localisation links für eine Eigenthümlichkeit der hysterischen Lähmung zu halten (Beweis: Die hysterischen Hemiplegien Briquet's l. 46, r. 14, die hysterischen Lähmungen Brown-Séquard's'): von 12197 linksseitige). Warum das so ist, wissen wir allerdings nicht. Am ersten könnte man sich vorstellen, dass unsere linke Körperhälfte als die weniger gebrauchte, geübte und gekräftigte leichter als die rechte (bezw. die entsprechende Grosshirnhälfte) einem krankhaften Process von der Art des hysterischen unterliegt. $\mathrm{Ob}$ man die weitere Ursache mit de Fleury ${ }^{2}$ ) in der verschiedenen Ernährung und Blutversorgung beider Hirnbälften, einem "geringeren Dynamismus" der rechten sehen will, muss um so mehr dem Belieben des Einzelnen überlassen bleiben, als wir hier von allen sicher constatirten Thatsachen weit entfernt sind.

Dass der Beginn der Atrophie zuerst an bestimmten Muskelgruppen sich zeigt (kleine Handmuskeln, Deltoideus), kommt vor, scheint aber nicht die Regel zu sein. Eher darf man ebenso wie für die bysterische (Babinski) auch für die cerebrale Form die gleichmässige Atrophie eines ganzen Abschnittes (Ober- oder Vorderarm) oder der ganzen Extremität als charakteristisch hinstellen. Das Bein und die Rumpfmaskeln betheiligen sich gewöhnlich nicht; einmal wurde Atrophie der Zunge, einmal gleichzeitige Atrophie der kleinen Handmuskeln der anderen Seite gesehen. Der Intensität nach scheint dieser Schwund nie so hohe Grade zu erreichen, wie der spinale (vergl. z. B. die Todd'schen Fälle), und dürfte dies Verhalten für die cerebrale (und hysterische) Form charakteristisch sein. Aber man darf auch nicht vergessen, dass die meisten Fälle sehr bald mit dem Tode endigten.

Wo die primäre Hirnaffection zur Heilung kam, verschwand auch die Muskelatrophie spurlos.

Einmal war ausser den Muskeln auch die Haut atrophisch; anch Störungen der Epidermisbildung, des Nägelwachsthums und andere trophische Störungen wurden als Complicationen beobachtet (Oedem, Hyperidrosis u. s. w.).

Die Sehnenreflexe waren gewöhnlich erhöht, selbst dann, wenn die Lähmung ausdrüeklich als schlaffe bezeichnet war. Die elek-

1) Citirt bei Bastian. Lancet 1874 .

2) Journal de Bordeaux 1869. - De l'hémiplégie hystérique etc. Bordeaux 1871. - Du dynamisme comparé des hémisphères du cerveau. Paris 1873 (ausführlich besprochen in Schmidt's Jahrbüchern).

Deutsche Zeitschr. f. Nervenheilkande. III. Bd. 
trische Erregbarkeit, die nur hie und da untersucht wurde, zeigte theils normales Verhalten, theils einfache Herabsetzung, einmal eine Steigerung und zweimal Aenderung des Zuckungscharakters (träge Zuckungen in den kleinen Handmuskeln).

Eine bestimmte Localisation des Gehirnherdes ist zum Zustandekommen der Atrophie nur insofern nöthig, als derselbe eben irgendwo innerhalb des motorischen Gebietes sitzen muss. Das ist Alles, was wir dariber sagen können, und ich denke auch nicht, dass Dark. schewits $\mathrm{ch}^{1}$ ) etwas Anderes meint, wenn er die Atrophie von der „besonderen Localisation" des Herdes abhängen lässt. Was iiber diese ganz allgemeine Bestimmung hinausgeht, ist haltlos. Luz z a to ${ }^{2}$ ) glaubt mit einer Igewissen Wahrseheinlichkeit aus dem Eintritt der Atrophie auf Erkrankung der Rinde schliessen zu dürfen. Nach unserer Zusammenstellung könnte er mit etwas mehr Recht das Gegentheil behaupten. Wir finden nämlich die Rinde erkrankt 7 mal, die tieferen Theile 9 mal, wohl beide zugleich 2 mal, und wenn wir die 2 Fälle Cantani's und Rosenthal's dazu nehmen, die Brïcke ebenfalls 2 mal. Warum überhaupt noch innerhalb der motorischen Bahn eine specielle Localisation nöthig sein sollte, lässt sich gar nicht einsehen, man mag taber das Zustandekommen der Atrophie eine Ansicht haben, welche man will.

Die Herde waren 8 mal durch Hämorrhagien, bezw. Thrombosen und Embolien entstanden, 5 mal waren es Tumoren (bezw. 7. Cantani und Rosenthal), und ebenfalls 5 mal Entzündungen und Abscesse, wobei ich die Fractur des Sebeitelbeins unter die Entzündungen stelle.

Die absteigende Degeneration fehlte von 11 darauf untersuchten Fällen 5 mal, also lange nicht so oft, wie Borgherini seiner Zeit annehmen musste, und ziehen wir hieraus wieder den Schluss, dass die absteigende Degeneration für das Zustandekommen der Atrophie in jeder Hinsicht völlig gleichgïltig ist.

Die peripheren Nerven wurden in den wenigen Fällen, wo sie uberhaupt untersucht wurden, normal befunden. An den Muskeln zeigte sich gewöhnlich einfache, ausnahmsweise (Roth und Muratow, Borgherini II) degenerative Atrophie.

Das nächste Ergebniss dieser Betrachtungen wäre, dass die Fälle von cerebraler Hemiplegie mit Atrophie sich in

1) Vortrag in der Gesellschaft der Moskauer Nerven- und Irrenärzte 17. Mai 1891. Arch. de neurol. XXIII. 1892. Mai.

2) Il sistema nervoso centrale. parte IIa. p. 595 (Biblioteca medica contemporanea Vallardi). 
nichts von den gewöhnlichen unterscheiden. Weder in der Localisation, noch in der Art des Krankheitsherdes noch in irgend einem etwa besonders constanten mehr nebensächlichen Moment bieten sie irgend eine Handhabe, die Atrophie an etwas Derartiges gebunden zu denken. Einzig und allein eben diese Atrophie ist es, was sie zu etwas Besonderem macht.

Nun erbebt sich die Frage: Sind hysterische, cerebrale und spinale posthemiplegische Muskelatrophien nur gradweise verschiedene Aeusserungen desselben Vorgangs im centralen Nervensystem, oder hat mindestens die letzte mit den beiden anderen ganz und gar nichts zu thun, während diese auf eine gemeinsame Ursache zurtickzuführen sind? Die Antwort darauf hängt nicht zum geringsten Theil von theoretischen Erwägungen ab. Aber ehe wir auf diese eingehen, dürfte es sich empfehlen, einmal einfach die Thatsachen neben einander za stellen.

Charakteristisch für die cerebrale (und hysterische) Form ist, dass sie sehr schnell eintritt, mehr gleichmässig einen ganzen $\mathrm{Ab}$ schnitt befällt und, nachdem sie eine gewisse Mittelstufe erlangt hat, keine Neigung mehr zum Weiterschreiten zeigt. Den ersten Zug muss man ohne Einschränkung gelten lassen, der zweite gilt schon nicht mehr allgemein, da auch cerebrale (und hysterische) Atrophien bei bestimmten Muskeln beginnen können und zwar bei den näm. lichen wie die spinalen. Gegen den dritten kann man immer den Vorbehalt machen, dass eine genügend lange beobachtete cerebrale Atrophie noch nicht existirt; entweder heilte sie oder trat der Tod ein. Mikroskopisch zeigt sich die cerebrale Atrophie fast immer als einfache, die spinale auch als degenerative; doch sind auch 2 Fälle degenerativer Atrophie bei der cerebralen Form beschrieben. Bezïglich der elektrischen Erregbarkeit haben sich bis jetzt keine fundamentalen Unterschiede ergeben. Die Entartungsreaction, die man nach Analogie mit der progressiven spinalen Muskelatrophie und der amyotrophischen Lateralsklerose bei der spinalen Form erwarten durfte, ist noch nicht nachgewiesen worden (die meisten Fälle überhaupt nicht elektrisch untersucht!). Andererseits ist es interessant, aus den Mittheilungen Eis enlohr's zu ersehen, dass auch bei cerebraler Atrophie Aenderungen des elektrischen Verhaltens vorkommen können, die Eisenlohr selbst zwar ausdrücklich nicht zur Entartungsreaction gerechnet wissen will, die aber doch an der Strasse dahin zu liegen scheinen. Und wenn man zu guter letzt an die Mittheilungen iber Entartungsreaction bei hysterischen Atrophien 
sich erinnert, muss einem der Versuch, elektrodiagnostische Unterschiede zwischen den verschiedenen Formen zu statuiren, wenn nicht überhaupt für verfehlt, doch zum wenigsten für verfrüht gelten. Auch von allen übrigen Symptomen ist keines für die eine oder andere Form charakteristisch.

Unter solchen Verhältnissen giebt den Ausschlag die Deutung der Befunde, die Theorie dieser Atrophien.

Charcot hält alle drei Formen für Folgen einer Erkrankung: desselben Organs, der Ganglienzellen der Vorderhörner. Auf folgende Weise: Die Fasern der Pyramidenbahnen bedürfen zur Erhaltung ihres normalen Lebens eines Einflusses, der von den Ganglienzellen der motorischen Gehirnpartien ausgeht. Fällt derselbe weg; entweder nach Zerstörung der Centren oder der Bahnen oder infolge der eigenthümlichen Erkrankung der Zellen, die der hysterischen Lähmung zu Grunde liegt, so greifen in den Fasern Vorgänge Platz, deren geringste Grade wir vorläufig noch nicht als krankhaftes Aussehen der Fasern kenntlich zu machen vermögen, deren höchste aber zu dem Bild der secundären Degeneration führen. Diese Vorgänge haben das Eigenthümliche, dass sie auf die Zellen der Vorderbörner, in denen die betreffenden Fasern endigen, als Reiz wirken. Die nächste Folge dieses Reizes ist eine erhöhte Erregbarkeit und Erregtheit der Ganglienzelle (erhöhte Reflexe, Muskelspannungen, Contracturen). Es kann aber auf dieses erste Stadium, das der Reizung, ein zweites der Ueberreizung oder Lähmung, im äussersten Fall der Tod der Zelle folgen, je nach der Widerstandsfähigkeit dieser, resp. der Stärke des Reizes verschieden bald und bei verschiedenen anatomischen Bildern, woriber das Schema auf S. 248, zu vergleichen. Die Folge dieser Lähmung, resp. des Todes der Zelle, ist die Muskelatrophie. Dieselbe ist gering und nur vorübergehend, wenn auch die Vorderhornzellen nur wenig oder vortbergehend ermidet sind, und sie erreicht die höchsten Grade und ist dauernd, wenn die Ueberreizung. den Tod der Zelle zur Folge gehabt hat. Diese Hypothese hat neben ihrer klaren Einheitlichkeit den grossen Vorzug, dass sie die Atrophie von der Erkrankung eines Organs abhängig macht, dessen Gesundheit nach zahllosen Erfahrungen zum normalen Leben der Muskeln unumgänglich nöthig ist. Sie leidet aber unter der Unsicherheit ihrer uncontrolirbaren Voraussetzungen und der einseitigen Betonung derselben, wobei sie ganz zu erwägen vergisst, ob nicht schon die einfache Abtrennung von den übergeordneten Centren auf die Vorderhörner schädlich einwirkt und nicht erst die secundär-degenerativen Processe. 
Davon wesentlich verschieden sind die Erklärungsversuche mittelst trophischer Hirncentren (S enator). Hier handelt es sich nur um die hysterische und cerebrale Form. Die spinale bleibt als den beiden anderen fremd gänzlich unberïcksichtigt. Die besagten Centren haben trophische Functionen entweder für die Vorderhornzellen des Rückenmarkes (Patella, Borgherini) oder direct für die Körpermusculatur (bes. deutsche Autoren) und liegen entweder in der motorischen Region (Patella) oder an irgend einer bis jetzt noch nicht entdeckten Stelle im Gehirn (Quincke, Hirt). Der von ihnen auf das untergeordnete Organ ausgetibte Einfluss, der unbekannter, vielleicht vasomotorischer ( $\mathrm{P}$ atella) Natur ist, wird entweder in den Pyramidenbahnen oder (Borgherini) irgendwo in den Hintersträngen in sensibeln oder besonderen trophischen Fasern herabgeleitet. Roth und Muratow betrachten die cerebralen Atrophien als Folge der Ausschaltung der vasomotorischen Rindencentren. Sie entstehen nach ihnen wie die chronisch-kachektischen Atrophien durch ungeniigenden Zufluss arteriellen Blutes, wobei nur noch zweifelhaft ist, $o b$ es sich dabei um einen primären Krampf der Vasoconstrictoren oder um eine Lähmung der Dilatatoren handelt. Diese Lehre von den trophischen Gehirncentren zeigt leider um so mehr Lücken und Widersprïche, je näher man sie betrachtet. Wir mögen diese Centren in einem Theil des Gehirns und ihre Bahnen im Rïckenmark unterbringen, wo immer wir wollen, stets wird sich eine so ïberwältigende Zahl negativer Fälle ergeben, dass rein statistisch geradezu unumstösslich bewiesen ist, dass trophische Gehirncentren allgemein wenigstens nicht existiren.

Hier kann man nicht zu einem Ziel kommen, wenn man die Augen stets nur auf unsere paar Ausnahmefälle gerichtet hält und nicht auf die Masse der so zu sagen normalen Vorgänge nach Gehirnläsionen. Diese Vorgänge lassen sich aber etwa folgendermassen charakterisiren: Bei Kindern sind Wachsthumsstörungen, resp. Atrophien $\left.{ }^{1}\right)$ central gelähmter Glieder ein zum mindesten häufiges Vorkommniss. Es hat aber deshalb, soviel ich weiss, noch Niemand jm kindlichen Gehirn nach trophischen Muskelcentren gesucht. Als

1) Dass die Volumsveränderung central gelähmter Glieder bei Kindern nicht immer und ausschliesslich ein passiver (Wachsthumshemmung), sondern auch ein activer (Atrophie) Vorgang ist, beweisen am schlagendsten die Fălle, wo zuletzt überhaupt kein normales Muskelgewebe mehr vorbanden ist. Aber auch dann, wenn die Differenz zwischen beiden Extremitäten in sebr kurzer Zeit (z. B. 3 Wochen) eine messbare Grösse erreicht, wird man Bedenken tragen, sie ganz auf Rechnung des gesunden, d. b. wachsendes Gliedes zu setzen. 
solche functioniren auch in den ersten Lebensjahren lediglich nur die grossen Ganglienzellen der Vorderhörner. Aber diese Zellen selbst sind nicht in der Lage, die Trennung vom übrigen Theil der corticomusculären Bahn ohne Beeinträchtigung ibrer Leistungsfähigkeit zu uberstehen, der Zusammenhang mit jenen ist ihnen zum normalen Leben unentbebrlich. Das ist aber nicht etwa eine Eigenthümlichkeit des Bewegungsapparates, sondern eine allgemeine Eigenschaft sämmtlicher Bahnen des kindlichen Nervensystems, die man mit Vortheil sogar in einer Untersuchungsmethode sich zu Nutze gemacht hat. Wenn wir nun sehen, dass bei Erwachsenen der Wegfall des vom Gehirn auf die Vorderhornzellen ausgeübten Einflusses für das regetative Leben der Muskeln ohne sonderliche Wirkung bleibt, so müssen wir daraus nothwendig schliessen, dass im Lauf der Jahre die Vorderhornzellen eine grössere Selbständigkeit erlangt haben, die sie befähigt, das von ihnen direct abhängige periphere Gebiet auch allein auf seinem normalen Zustand zu erhalten. Und wiederum ist das nicht etwas auf die Vorderhornzellen Beschränktes, sondern ein fir das ganze Nervensystem zutreffendes Ergebniss seiner Entwicklung und jahrelangen Thätigkeit. Aber der Grad dieser Selbständigkeft ist ein individuell verschiedener; im Allgemeinen ziemlich gross, so dass Abnormitäten kaum zu merken sind, wobei man aber nicht vergessen darf, dass das Fehlen trophischer Störungen bei centraler Lähmung nur mit Riucksicht auf die raschen und bedeutenden Veränderungen bei der peripheren gemeint ist, und dass Atrophien geringeren Grades, z. B. an den kleinen Handmuskeln (Eisenlohr) bei Hemiplegischen, als Zeichen doch vorhandener Störung nicht zu den Seltenheiten gehören. Hie und da nun geschieht es, dass auch einmal bei Erwachsenen der Wegfall des vom Gehirn ausgehenden Reizes stärkere Abmagerung der Muskeln zur Folge hat, ein Beweis, dass ganz vereinzelt die Vorderhornzellen über die Zeit hinaus, eventuell für immer in jenem Abhängigkeitsverhältniss vom Gehirn bleiben, das für das Kindesalter charakteristisch ist. Es sind also unsere cerebralen und hysterischen posthemiplegischen Atrophien im Grunde identiseh mit den Wachsthumsstörungen und Atrophien bei centralen Lähmungen im Kindesalter, beide der Ausdruck einer geringeren Autonomie der secundären Centren. Damit stimmt das Bild derselben gut überein. Ihr schnelles Erscheinen, die im Allgemeinen mässige Intensität (da ihnen ja nicht eine schwerere Störung, sondern nur eine gewisse Schwäche, ein verminderter Tonus der Vorderhornzellen zu Grunde liegt), ihr spärliches Verschwinden, wenn dieser Tonus wieder eintritt. Von der spinalen 
Form sind sie insofern wesentlich verschieden, als sie ein nothwendiges Ergebniss der Gehirnläsion im gegebenen Fall darstellen, während man die spinale mehr als eine zufällige Complication des in seiner Deutung noch etwas zweifelhaften Processes der secundären Degeneration betrachten möchte. Dass sie trotzdem mit derselben soweit Gemeinsames haben, rîhrt daher, dass sie eben alle drei Folge der herabgesetzten, bezw. aufgehobenen Thätigkeit desselben Zellcomplexes sind.

Eine wirkliche Erklärung ist damit freilich nicht gegeben. Aber eine solche ist einstweisen überhaupt unmöglich in einer Frage, wo nicht nur die Voraussetzungen schwankend und unsicher, sondern auch die Thatsachen' spärlich und weit von einander stehend gefunden werden. Es genügt, wenn klar geworden ist, dass es sich in unseren Fällen nicht um Erscheinungen handelt, die ohne Weiteres zur Erschliessung allgemeiner Eigenschaften unseres centralen Nervensystems benutzt werden dürfen, dass dieselben vielmehr als Ausnahmen und Abweichungen von einer immer und immer wieder bestätigten Regel zu behandeln sind.

Herrn Geheimrath A rnold und Herrn Prof. Hoffmann bin ich für das Interesse, das sie dieser Arbeit entgegengebracht haben, zu grossem Dank verpflichtet. 